\title{
BeppoSAX observations of CF Tucanae and TY Pyxidis
}

\author{
E. Franciosini ${ }^{1}$, R. Pallavicini ${ }^{1}$, and G. Tagliaferri ${ }^{2}$ \\ 1 INAF/Osservatorio Astronomico di Palermo, Piazza del Parlamento 1, 90134 Palermo, Italy \\ 2 INAF/Osservatorio Astronomico di Brera, via E. Bianchi 46, 23807 Merate (LC), Italy
}

Received 24 September 2002 / Accepted 14 November 2002

\begin{abstract}
We present the results of BeppoSAX observations of the RS CVn binary systems CF Tuc and TY Pyx. A longduration flare was detected on CF Tuc just at the beginning of the observation; a second smaller flare occurred about half a period later. The light curve of TY Pyx shows a significant orbital modulation of the quiescent emission with possible evidence for eclipses, and strong flares. We find that the coronae of the two stars are very similar: both systems have quiescent temperatures of $\sim 10$ and 20-25 MK, with only a factor of two difference in the emission measures. During the flares, temperatures of $\sim 30-$ $40 \mathrm{MK}$ are reached; these temperatures are not high enough to give detectable hard X-ray emission in the PDS. Both stars have subsolar coronal metallicities: we confirm the very low coronal abundance of CF Tuc $\left(Z \sim 0.1-0.2 Z_{\odot}\right)$, while for TY Pyx we find $Z \sim 0.5-0.6 Z_{\odot}$. For both stars the coronal metallicities are in good agreement with their measured photospheric abundances.
\end{abstract}

Key words. stars: binaries: close - stars: activity - stars: coronae - stars: individual: CF Tuc - stars: individual: TY Pyx X-rays: stars

\section{Introduction}

RS CVn close binaries are among the most active late-type stars, showing strong coronal emission, with quiescent $\mathrm{X}$-ray luminosities $L_{X} \sim 10^{30}-10^{31} \mathrm{erg} \mathrm{s}^{-1}$ (Dempsey et al. 1993), and frequent flares, similar to those observed on the Sun but on much larger scales. Hard X-ray emission up to $\sim 50 \mathrm{keV}$ has been detected by BeppoSAX during large flares on the RS CVn system UX Ari (Franciosini et al. 2001), as well as on Algol (Favata \& Schmitt 1999) and on the young rapidly-rotating single star AB Dor (Pallavicini \& Tagliaferri 1999; Pallavicini 2001). ASCA and BeppoSAX observations have shown that many active binaries have coronae with subsolar metal abundances, which in some cases are also lower than their photospheric abundances (e.g. II Peg, Covino et al. 2000). In this paper we present the results of BeppoSAX observations of the two RS CVn systems CF Tucanae and TY Pyxidis.

CF Tucanae (HD 5303) is a partially eclipsing RS CVn binary consisting of a G0 V primary and a K4 IV secondary, with an orbital period of 2.8 days and an inclination $i=64^{\circ}$; the distance from Hipparcos is $d=86$ pc (ESA 1997), higher than the previously assumed distance of $54 \mathrm{pc}$. This star, first discovered as an X-ray source by HEAO-1 (Walter et al. 1980), was observed by the Einstein IPC with a luminosity $L_{X} \sim$ $3 \times 10^{30} \mathrm{erg} \mathrm{s}^{-1}$ (Walter \& Bowyer 1981; Drake et al. 1992) and later by ROSAT both during the All-Sky Survey (RASS) and in a PSPC pointed observation. During the RASS it showed a

Send offprint requests to: E. Franciosini, e-mail: francio@astropa.unipa.it variable X-ray emission between 1.8 and 5.6 cts s$^{-1}$ (Kürster \& Dennerl 1993; Dempsey et al. 1993), with a minimum around orbital phase 0.6 , which was interpreted as rotational modulation induced by two active regions, associated with the photospheric spots on the active $\mathrm{K}$ star derived from simultaneous Doppler imaging (Kürster \& Dennerl 1993). The pointed PSPC observation caught one of the most energetic and longest stellar X-ray flares ever observed (Kürster \& Schmitt 1996). The $\mathrm{X}$-ray emission increased by a factor of $\sim 10$ in 1.5 days, and decayed slowly, returning to the quiescent level 7 days after the peak. The peak temperature was $\sim 7 \times 10^{7} \mathrm{~K}$. The light curve did not show any evidence of eclipses or rotational modulation, implying an extended source region, or a compact source near the stellar poles. Clear evidence for rotational modulation of the quiescent emission has been observed also by EUVE (Schmitt et al. 1996; Gunn et al. 1997; Osten \& Brown 1999) and at radio wavelengths (Gunn et al. 1997; Budding et al. 1999), with the UV and radio light curves anticorrelated with the optical light curve.

An interesting characteristic of CF Tuc is its low metal abundance. Randich et al. (1993) measured the photospheric iron abundance, finding $[\mathrm{Fe} / \mathrm{H}]=-0.5$ (i.e. $\sim 0.32$ solar) for the $\mathrm{G}$ star and $[\mathrm{Fe} / \mathrm{H}]=-0.9(\sim 0.13$ solar $)$ for the $\mathrm{K}$ star. Schmitt et al. (1996), by comparing EUVE observations with the ROSAT PSPC data from Kürster \& Schmitt (1996), concluded that the coronal metallicity is most probably $\sim 0.1$ solar.

TY Pyxidis (HD 77137) is an eclipsing RS CVn binary system $\left(i=88^{\circ}\right)$ consisting of two nearly equal G5 IV stars orbiting each other with a period of 3.2 days, and located at 
a distance of 56 pc (ESA 1997). This system was observed for a full orbital period by EXOSAT (Culhane et al. 1990). During the observation strong flares were detected; both primary and secondary eclipses were evident in the $0.05-2.0 \mathrm{keV}$ band, but were not observed at higher energies, implying a more extended distribution of the higher temperature component. Detailed modeling of the light curve suggested an emission region located between the two stars (Pres et al. 1995). TY Pyx has been observed later by ROSAT during the RASS: in this case the source showed a constant count rate of $1.9 \mathrm{cts} \mathrm{s}^{-1}$ (Dempsey et al. 1993; Neff et al. 1996).

The photospheric iron abundance of TY Pyx has been measured by Randich et al. (1993), who derived a value of $[\mathrm{Fe} / \mathrm{H}]=-0.2(\sim 0.63$ solar $)$ for both stars.

The paper is organized as follows. Obervations and data analysis are described in Sect. 2; in Sect. 3 we present the results. Discussion and conclusions are given in Sect. 4.

\section{Observations and data analysis}

Both binaries were observed by BeppoSAX using the LECS (0.1-10 keV), the two available MECS (1.6-10 keV) and the PDS (15-300 keV) instruments.

The observation of CF Tuc was performed from 03:07 UT on July 18,2000 to $18: 35$ UT on July 21,2000 , for a total duration of $315 \mathrm{ksec}$, corresponding to $\sim 1.3$ orbital periods. The effective exposure times were 68,161 and $177 \mathrm{ksec}$ in the LECS, MECS and PDS, respectively.

TY Pyx was observed twice, in December 2000 and in May 2001. The first observation started at 21:00 UT on December 14, 2000 and ended at 12:12 UT on December 19, 2000 , after $400 \mathrm{ksec}$ of elapsed time, i.e. $\sim 1.4$ orbital periods. The total effective exposure times were 68, 132 and $129 \mathrm{ksec}$ for the LECS, MECS and PDS, respectively. The second observation was performed from 18:58 UT on May 9, 2001 to 11:35 UT on May 11, 2001, lasting $144 \mathrm{ksec}$, i.e. half an orbital period. Effective LECS, MECS and PDS exposure times were 22,61 and $60 \mathrm{ksec}$, respectively.

The lower exposure times in the LECS are due to the instrument being operated only in Earth shadow, thus reducing its observing efficiency.

The LECS and MECS data analysis was based on the linearized and cleaned event files obtained from the BeppoSAX Science Data Center (SDC) on-line archive. Spectra and light curves were accumulated using the XSELECT v.1.4b software in the FTOOLS v4.2 package, using an extraction region of 8 and 4 arcmin radius for the LECS and MECS, respectively; the chosen regions provide more than $90 \%$ of the source counts at all energies for both instruments. LECS and MECS background spectra accumulated from blank field exposures and available at the SDC were used for spectral analysis. For the PDS, both the light curve and the spectrum were retrieved from the SDC on-line archive. For both stars however no significant emission was detected by the PDS instrument, and therefore we will not consider it further.

The spectral analysis was performed with the XSPEC v11.0 package, using the response matrices released by the SDC in September 1997 for the MECS and in January 2000 for the
LECS. The spectra were rebinned in order to have at least 30 counts per bin. The LECS data have been fitted in the $0.1-$ $4 \mathrm{keV}$ band due to calibration problems at higher energies (Fiore et al. 1999). The spectral fits were performed using $\chi^{2}$ statistics with weights evaluated according to Gehrels (1986). In order to account for the uncertainties in the intercalibration of the LECS and MECS instruments, a relative normalization factor (which was left free to vary) has been included in the fits: the derived best-fit values are between 0.74 and 0.86 , in agreement with the allowed range of 0.7-1 (Fiore et al. 1999). All spectra have been fitted assuming an optically-thin thermal plasma model with two discrete temperature components, using the MEKAL emissivities (Mewe et al. 1995) with variable global metal abundances, and taking into account the effect of interstellar absorption. The interstellar column density $N_{\mathrm{H}}$ was initially left as a free parameter: the derived best-fit values are consistent with those expected for the distance of CF Tuc and TY Pyx assuming an interstellar hydrogen volume density of $0.07 \mathrm{~cm}^{-3}$ (Paresce 1984), i.e. $N_{\mathrm{H}}=2 \times 10^{19} \mathrm{~cm}^{-2}$ for CF Tuc and $N_{\mathrm{H}}=1.2 \times 10^{19} \mathrm{~cm}^{-2}$ for TY Pyx, but they are poorly constrained by the fit. We therefore repeated the fits keeping $N_{\mathrm{H}}$ fixed to the expected values. In all spectral fits we have chosen to consider $90 \%$ confidence errors for the three interesting parameters $T_{1}, T_{2}$ and $Z / Z_{\odot}$.

\section{Results}

\subsection{CF Tucanae}

The LECS and MECS light curves of CF Tuc are shown in Fig. 1. A moderate, long-duration flare was detected just at the beginning of the observation. The count rate increased by a factor 2.5 in 5 hours and returned to a quiescent level after 17 hours. The increase in the count rate is coincident with the time of secondary eclipse (eclipse of the active $\mathrm{K}$ star); however, having no data before it, we cannot distinguish whether this increase might be due to an eclipse of the flaring region rather than to the rising phase of the flare or to both. A second smaller flare occurred about half a period later; this flare had a rise time of $\sim 1.7$ hours and a decay to the quiescent level $\$ 10$ hours, with a factor $\sim 2$ increase of the count rate. During the second half of the observation the star remained at a quiescent level; some low-level variability is present, but we find no evidence of rotational modulation of the X-ray emission. However, the available quiescent data cover only a fraction of $\sim 0.6$ of the orbital period, therefore we cannot exclude that some modulation might indeed be present. There is also no evidence of secondary eclipse in the quiescent data.

We have performed spectral analysis of the LECS and MECS data in four separate time intervals covering the two flares and the quiescent emission; the chosen intervals are indicated in the bottom panel of Fig. 1. For the second flare, where the number of counts is low, we kept the low temperature component fixed to its quiescent value, since it was not constrained by the fit. The best-fit parameters are given in Table 1 . The spectra of the quiescent emission and at the peak of the first flare are shown in Fig. 2 together with the corresponding best-fit models. During quiescence we find temperatures 
Table 1. Best-fit parameters of the 2-T models for CF Tuc. Errors are $90 \%$ confidence ranges for three interesting parameters.

\begin{tabular}{lccccccccr}
\hline \hline & $Z / Z_{\odot}$ & $\begin{array}{c}k T_{1} \\
(\mathrm{keV})\end{array}$ & $\begin{array}{c}k T_{2} \\
(\mathrm{keV})\end{array}$ & $\begin{array}{c}E M_{1} \\
\left(10^{53} \mathrm{~cm}^{-3}\right)\end{array}$ & $\begin{array}{c}E M_{2} \\
F_{0.1-10 \mathrm{keV}}^{a} \\
\left(10^{-11} \mathrm{erg} \mathrm{cm}^{-2} \mathrm{~s}^{-1}\right)\end{array}$ & $\begin{array}{c}F_{0.1-2 . \mathrm{keV}}^{a} \\
\chi_{\mathrm{r}}^{2}\end{array}$ & d.o.f. \\
\hline$q$ & $0.12_{-0.07}^{+0.18}$ & $0.79_{-0.40}^{+0.38}$ & $2.09_{-0.35}^{+1.23}$ & 3.4 & 5.5 & 1.1 & 0.9 & 0.56 & 122 \\
peak1 & $0.11_{-0.11}^{+0.26}$ & $1.83_{-0.56}^{+1.64}$ & $3.21_{-0.55}^{+0.75}$ & 4.5 & 10.5 & 2.1 & 1.4 & 0.62 & 87 \\
dec1 & $0.23_{-0.18}^{+0.34}$ & $0.71_{-0.52}^{+0.93}$ & $2.08_{-0.26}^{+0.32}$ & 1.7 & 9.8 & 1.5 & 1.2 & 0.51 & 69 \\
fl2 ${ }^{b}$ & $0.15_{-0.15}^{+0.19}$ & 0.80 & $2.91_{-0.53}^{+0.75}$ & 3.4 & 9.4 & 1.7 & 1.3 & 0.50 & 44 \\
\hline
\end{tabular}

${ }^{a}$ Unabsorbed X-ray flux in the $0.1-10 \mathrm{keV}$ band and in the ROSAT $(0.1-2.4 \mathrm{keV})$ band.

${ }^{b}$ The first component was kept fixed to the quiescent value since it was not constrained by the fit. In this case errors are computed for two interesting parameters.

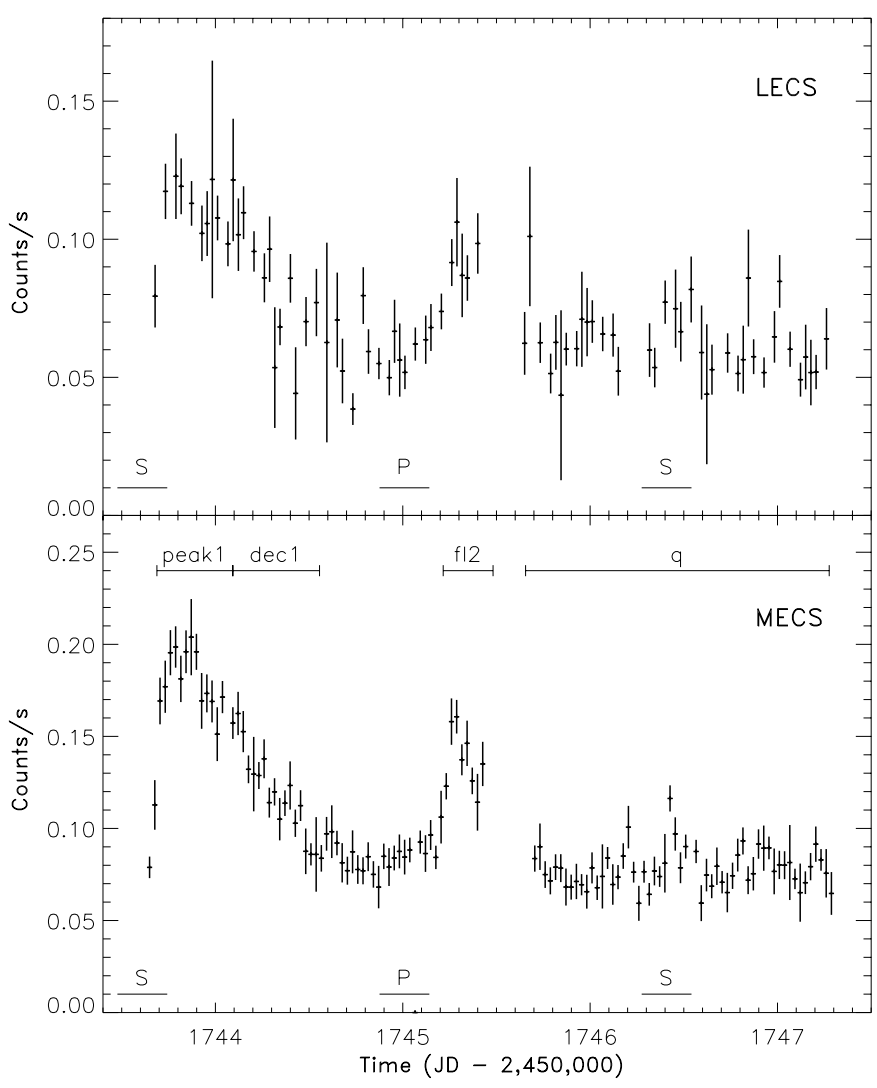

Fig. 1. LECS and MECS light curves of CF Tuc in July 2000. Data are binned over $2400 \mathrm{~s}$. The lines marked with $\mathrm{P}$ and $\mathrm{S}$ indicate the intervals corresponding to primary and secondary eclipses respectively, computed according to the ephemeris HJD $=2445606.9165+$ $2.797672 E$ by Budding (1985). The estimated uncertainty in the eclipse timing is $\sim 0.02^{\mathrm{d}}$. The intervals in which spectral analysis has been performed are indicated in the bottom panel (labels correspond to those in Table 1).

of $\sim 9$ and $\sim 24 \mathrm{MK}$, with emission measures of $3 \times 10^{53}$ and $5 \times 10^{53} \mathrm{~cm}^{-3}$, respectively. The luminosity in the $0.1-10 \mathrm{keV}$ band is $L_{\mathrm{X}} \sim 9 \times 10^{30} \mathrm{erg} \mathrm{s}^{-1}$. Both temperatures are significantly higher at the peak of the first flare compared to the quiescent and decay spectra, reaching values of $T \sim 21$ and $\sim 37$ MK; the peak luminosity is $L_{\mathrm{X}} \sim 2 \times 10^{31} \mathrm{erg} \mathrm{s}^{-1}$. During the second flare the higher temperature component increases to $T_{2} \sim 34 \mathrm{MK}$.
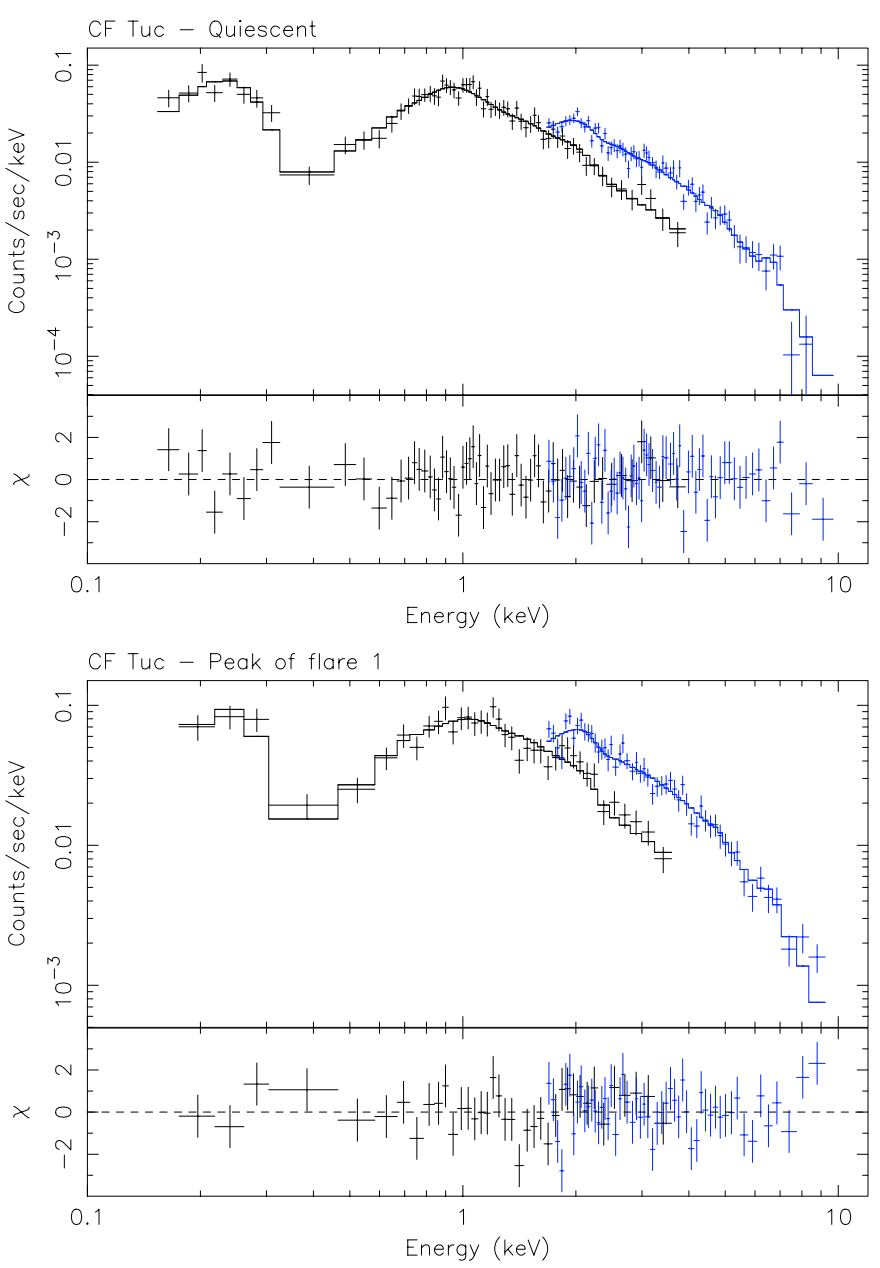

Fig. 2. LECS and MECS spectra of CF Tuc obtained during quiescence (top panel) and at the peak of the first flare (bottom panel). The best-fit model is also shown.

We find that the best fit metallicity is $Z \sim 0.1-0.2 Z_{\odot}$, thus confirming the previous ROSAT and EUVE results (Schmitt et al. 1996). The coronal metallicity is also consistent with the low photospheric abundance derived by Randich et al. (1993).

\subsection{TY Pyxidis}

The LECS and MECS light curves of TY Pyx for both observations are shown in Fig. 3. In both cases the star was 

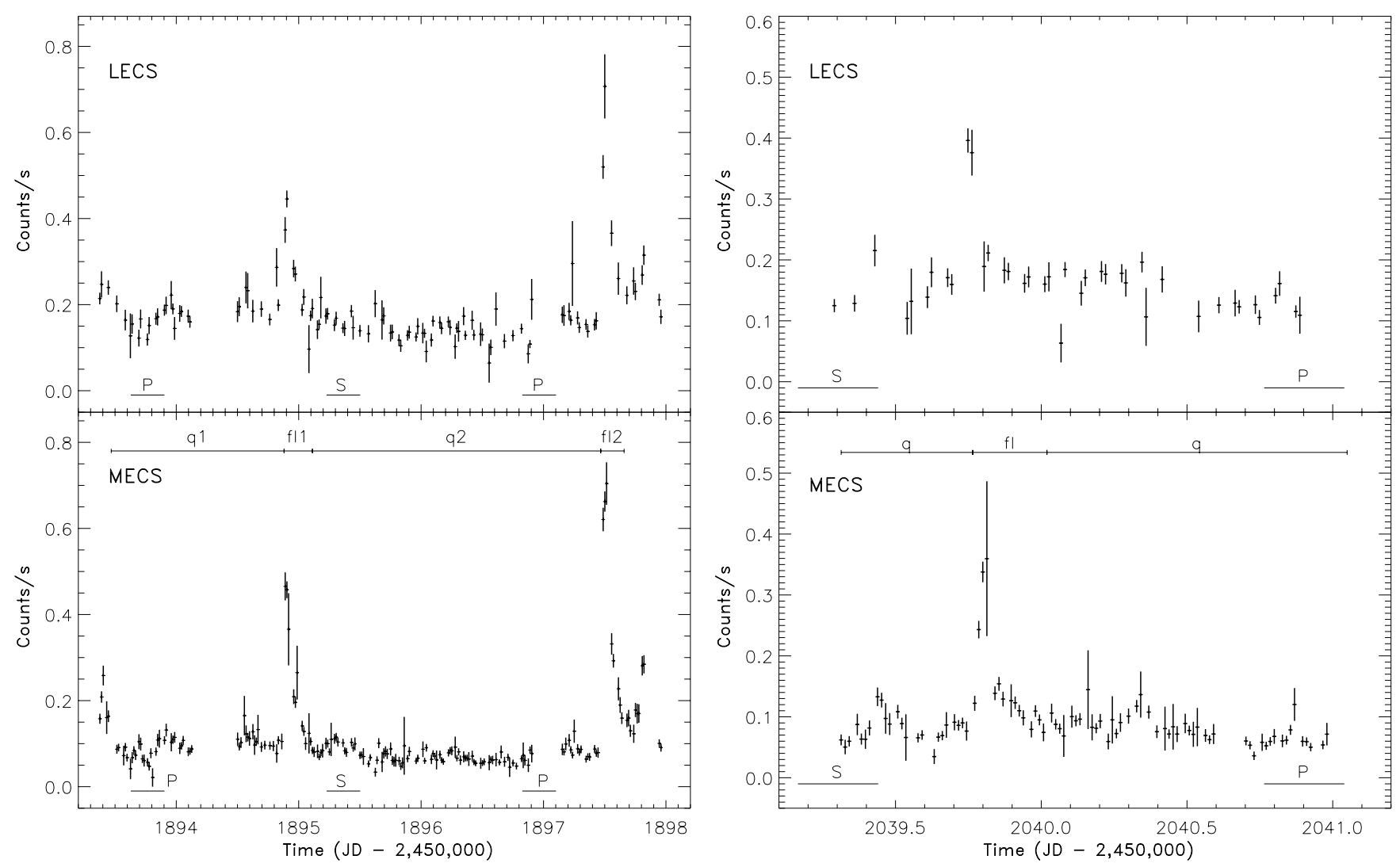

Fig. 3. LECS and MECS light curves of TY Pyx observed in Dec. 2000 (left panels) and in May 2001 (right panels). Data are binned over 1200 s. Note the different scales (in abscissa and ordinate) for the two panels. The lines marked with P and S indicate (with an estimated uncertainty of $\sim 0.02^{\mathrm{d}}$ ) the intervals corresponding to primary and secondary eclipses respectively, computed according to the ephemeris HJD = $2443548.66956+3.198581 E$ by Andersen et al. (1981). The intervals in which spectral analysis has been performed are also indicated (labels correspond to those in Table 2).

Table 2. Best-fit parameters of the 2-T models for TY Pyx. Errors are 90\% confidence ranges for three interesting parameters.

\begin{tabular}{lccccccccr}
\hline \hline & $Z / Z_{\odot}$ & $\begin{array}{c}k T_{1} \\
(\mathrm{keV})\end{array}$ & $\begin{array}{c}k T_{2} \\
(\mathrm{keV})\end{array}$ & $\begin{array}{c}E M_{1} \\
\left(10^{53} \mathrm{~cm}^{-3}\right)\end{array}$ & \begin{tabular}{c}
$E M_{2}$ \\
\multicolumn{7}{c}{ December 2000 } \\
$\left(10^{-11} \mathrm{erg} \mathrm{cm}{ }^{-2} \mathrm{~s}^{-1}\right)$
\end{tabular} \\
\hline$q 1$ & $0.56_{-0.24}^{+0.44}$ & $0.83_{-0.21}^{+0.22}$ & $2.06_{-0.40}^{+1.88}$ & 1.9 & 2.7 & 2.0 & 1.7 & 0.75 & 108 \\
$q 2$ & $0.57_{-0.19}^{+0.36}$ & $0.77_{-0.14}^{+0.08}$ & $2.02_{-0.30}^{+0.45}$ & 1.7 & 2.3 & 1.8 & 1.6 & 0.72 & 156 \\
$\mathrm{fl} 1$ & $0.76_{-0.43}^{+0.80}$ & $0.80_{-0.16}^{+0.20}$ & $3.01_{-0.61}^{+1.64}$ & 1.8 & 5.4 & 3.6 & 2.7 & 0.80 & 72 \\
$\mathrm{fl} 2^{b}$ & $0.62_{-0.27}^{+0.34}$ & 0.80 & $3.58_{-0.49}^{+0.71}$ & 1.8 & 10.9 & 5.6 & 3.6 & 0.63 & 63 \\
& & & & May 2001 & & & & \\
$q$ & $0.62_{-0.25}^{+0.52}$ & $0.80_{-0.14}^{+0.10}$ & $2.20_{-0.37}^{+0.78}$ & 1.6 & 2.1 & 1.7 & 1.0 & 0.64 & 114 \\
$\mathrm{fl}^{b}$ & $0.67_{-0.20}^{+0.26}$ & 0.80 & $2.62_{-0.37}^{+0.49}$ & 1.6 & 6.6 & 2.7 & 2.2 & 0.60 & 67 \\
\hline
\end{tabular}

${ }^{a}$ Unabsorbed X-ray flux in the $0.1-10 \mathrm{keV}$ band and in the ROSAT $(0.1-2.4 \mathrm{keV})$ band.

${ }^{b}$ The first component was kept fixed to the quiescent value in order to better constrain the parameters. In this case confidence ranges are computed for two interesting parameters.

very active, showing strong flares, with increases in the count rate up to factors of 10 , and typical rise and decay times of $\$ 1$ hour and $\sim 3-4$ hours, respectively. The quiescent emission (i.e. outside obvious flares) shows significant variability, but the average level of the emission is the same for both observations. We have folded the December 2000 quiescent light curve (segments labelled $q 1$ and $q 2$ in Fig. 3) with the orbital period, using the ephemeris HJD $=2443548.66956+3.198581 E$ given by Andersen et al. (1981). The result is shown in Fig. 4: the quiescent emission is clearly modulated with the orbital period, with higher count rates during phases $0-0.5$. The MECS light curve shows a small dip around the time of primary 

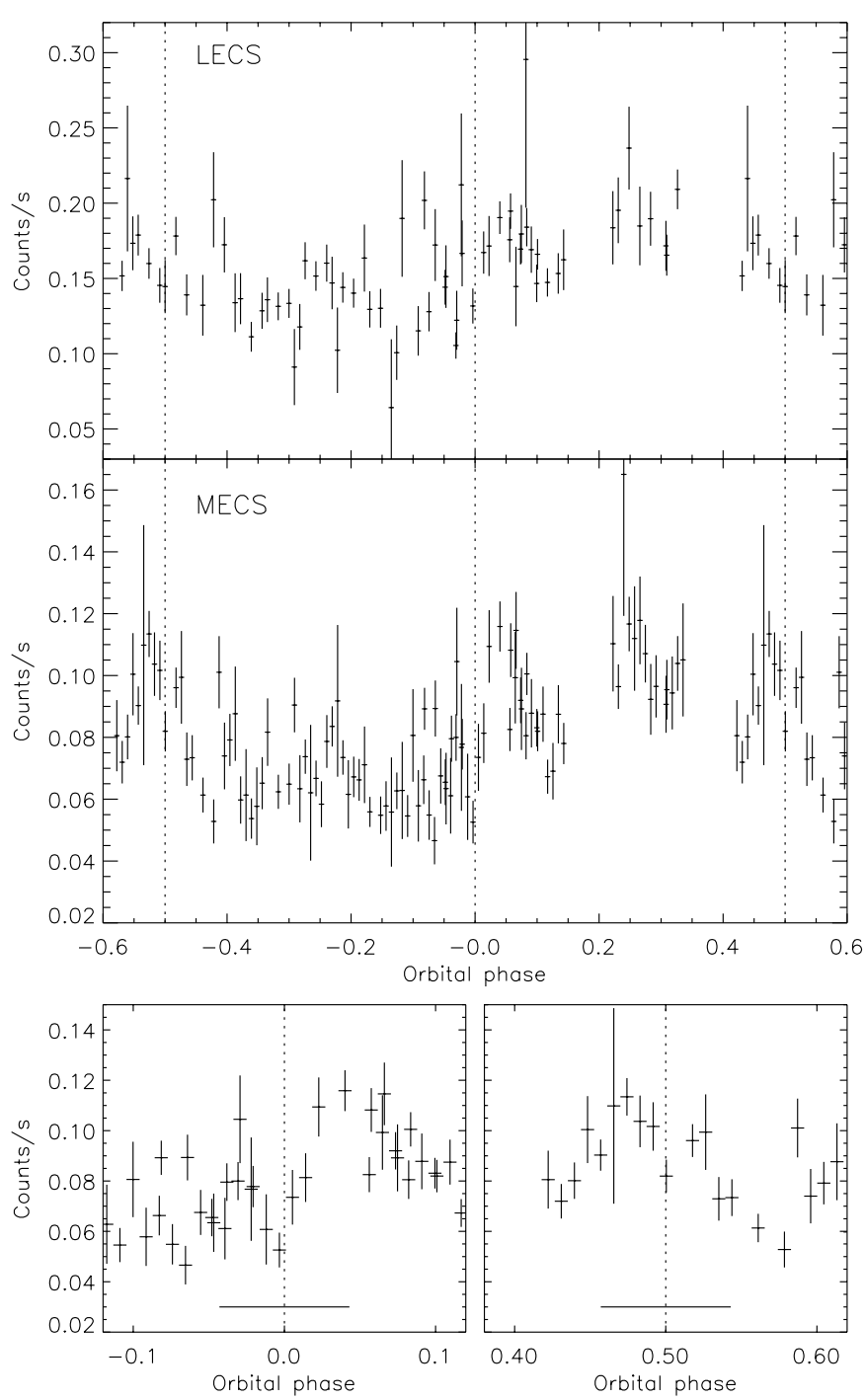

Fig. 4. Top panels: LECS and MECS quiescent light curves of TY Pyx in December 2000 folded with the orbital period, using the ephemeris by Andersen et al. (1981). Data are binned over $2400 \mathrm{~s}$. Vertical dotted lines mark the center of primary and secondary eclipses (phases 0 and 0.5; see Fig. 3). Bottom panels: expanded view of the folded MECS light curve around primary and secondary eclipses. The bars at the bottom indicate the duration of each eclipse.

eclipse and possibly at secondary eclipse (see bottom panels of Fig. 4); while the dip around primary eclipse is statistically significant, the one at secondary eclipse is not, the variation in the count rate being below $2 \sigma$. There is no evidence of eclipses in the May 2001 observation (Fig. 3).

We have performed spectral analysis of the quiescent emission and of the strongest flares observed both in December 2000 and May 2001. The chosen intervals are indicated in Fig. 3: for the December 2000 observation we analysed separately the quiescent emission before and after the first strong flare, while for the May 2001 observation the quiescent intervals before and after the flare were summed together. In the case of the second flare of December 2000 and of the flare of May 2001 we kept the low temperature component fixed to the quiescent value, in order to better constrain the fit. The best-fit
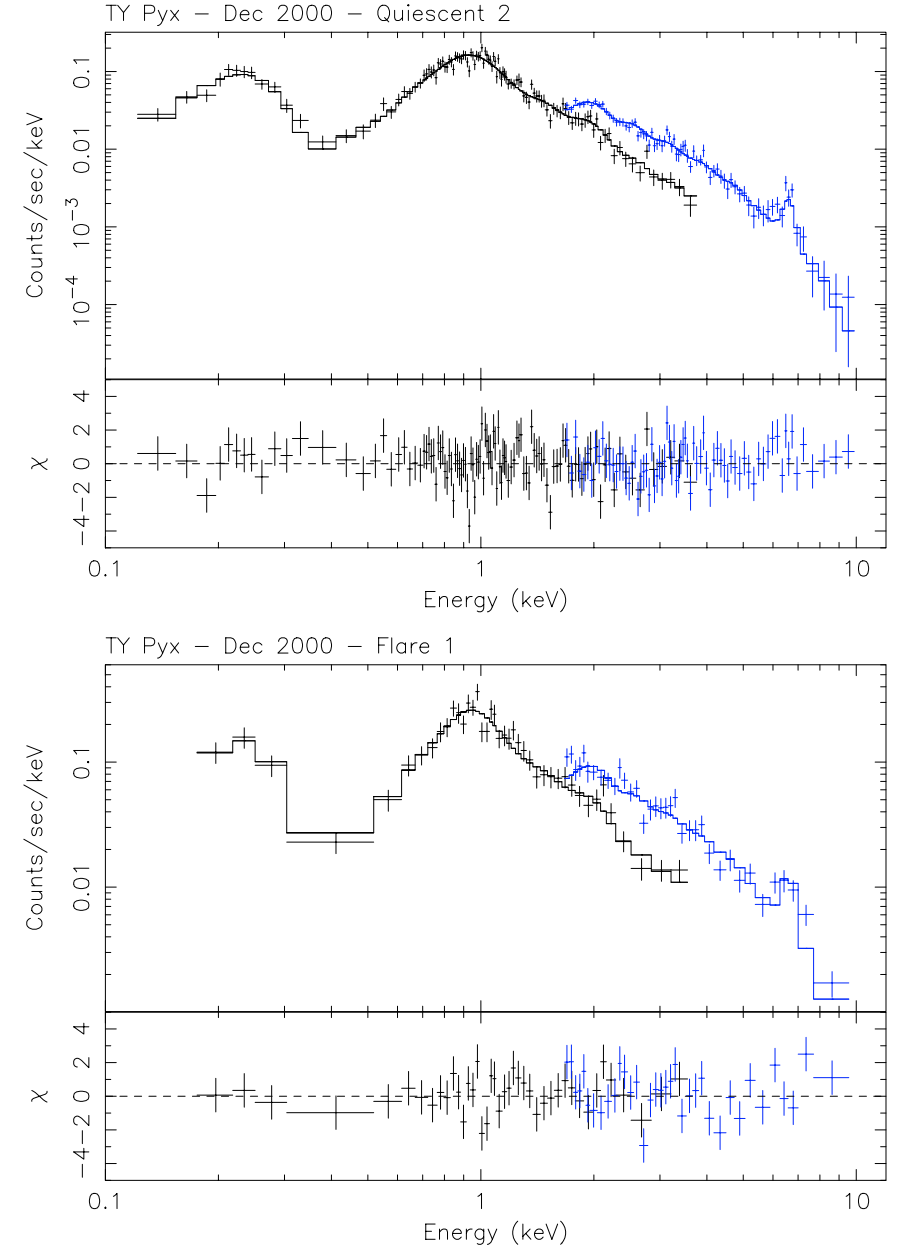

Fig. 5. LECS and MECS spectra of TY Pyx obtained in December 2000 during the quiescent period after the first flare (top panel) and during the first flare (bottom panel). The best-fit model is also shown. Note the stronger $\mathrm{Fe} \mathrm{K}$ line at $6.7 \mathrm{keV}$ with respect to the CF Tuc spectra in Fig. 2.

parameters are given in Table 2. In Fig. 5 we show two examples of quiescent and flare spectra, from the December 2000 observation, together with the best-fit models.

The best-fit parameters of the quiescent emission are very similar in both observations, indicating that the corona of TY Pyx is quite stable over several months. We find temperatures of $\sim 9-10 \mathrm{MK}$ and 23-25 MK, with emission measures of $\sim(1.6-1.9) \times 10^{53}$ and $\sim(2.1-2.7) \times 10^{53} \mathrm{~cm}^{-3}$, respectively. Note that the temperatures are very similar to those found for CF Tuc, while the emission measures are lower by a factor of $\sim 2$. The quiescent X-ray luminosity in the $0.1-10 \mathrm{keV}$ band is $L_{X} \sim 7 \times 10^{30} \mathrm{erg} \mathrm{s}^{-1}$. During the flares, temperatures up to $\sim 40 \mathrm{MK}$ are reached, with peak luminosities of $\sim 2 \times 10^{31} \mathrm{erg} \mathrm{s}^{-1}$.

We find that the coronal metallicity of TY Pyx is $Z \sim$ $0.5-0.7 Z_{\odot}$. This value is consistent with the measured iron photospheric abundance of 0.63 solar (Randich et al. 1993). 


\section{Discussion and conclusions}

In this paper we have presented the results of BeppoSAX observations of the two RS CVn binaries CF Tuc and TY Pyx. Both systems show high erratic variability with frequent flares detected.

Spectral analysis indicates that there is a very close similarity between the coronae of CF Tuc and TY Pyx. The quiescent emission from both binaries is reproduced by two components with temperatures of $\sim 10$ and $\sim 24 \mathrm{MK}$, with only a small difference in the emission measures (by a factor of $\sim 2$ ). The corona of TY Pyx appears also to be quite stable, with nearly the same values of temperature and emission measure over a time scale of five months.

During flares, temperatures of $\sim 30-40 \mathrm{MK}$ are reached in both binaries. These temperatures are consistent with the fact that there is no evidence of hard X-ray emission in the PDS instrument. In fact, hard X-rays from stars have been detected by BeppoSAX only during the rise phase and at the peak of large flares on Algol (Favata \& Schmitt 1999), AB Dor (Pallavicini \& Tagliaferri 1999) and UX Ari (Franciosini et al. 2001), when temperatures in excess of $100 \mathrm{MK}$ were reached; the hard Xray emission can be attributed to the thermal emission from such hot plasma (Pallavicini 2001). At the temperatures of the flaring plasma observed on CF Tuc and TY Pyx, the emission is not sufficiently strong to give a detectable signal in the PDS.

The low count rate of both stars and the modest intensity of the detected flares does not allow us to perform a detailed time-dependent spectral analysis of the flares, thus preventing the study of the flare evolution using available flare models (e.g. Maggio et al. 2000).

Both stars have subsolar coronal metallicities, as commonly found for most of the active stars observed with ASCA and BeppoSAX. For CF Tuc we find $Z \sim 0.1-0.2 Z_{\odot}$, which confirms the very low abundance $(\sim 0.1$ solar $)$ suggested by EUVE and ROSAT data (Schmitt et al. 1996); the values derived from our observation are also in agreement with the photospheric abundance of the $\mathrm{K}$ star ( 0.13 solar) measured by Randich et al. (1993). TY Pyx has a metallicity $Z \sim 0.5-0.7 Z_{\odot}$ which is also consistent with its photospheric abundance (Randich et al. 1993). Thus the low coronal abundances of CF Tuc and TY Pyx simply reflects their low photospheric abundances. Similar results have been obtained for several other stars observed by ASCA and BeppoSAX (e.g. Ortolani et al. 1997; Favata et al. 1997a,b; Maggio et al. 1998; Tagliaferri et al. 1999); however there are cases, such as AB Dor (Mewe et al. 1996; Maggio et al. 2000; Güdel et al. 2001a) or II Peg (Covino et al. 2000), where the coronal metallicity is found to be significantly lower than the measured photospheric value: in this case, the observed difference between the coronal and photospheric abundance has been attributed to fractionation induced by magnetic fields in active stars. We do not find evidence, in either binary, for significant variations of the coronal abundance during flares with respect to the quiescent value, contrary to what observed in other stars, such as Algol (Ottmann \& Schmitt 1996; Favata \& Schmitt 1999), II Peg (Mewe et al. 1997), EV Lac (Favata et al. 2000), or YY Gem (Tagliaferri et al. 2001; Güdel et al. 2001b), where abundance increases up to a factor of 5 have been reported during strong flares.

The quiescent emission of TY Pyx is modulated with the orbital period, with higher emission level between phases 0 and 0.5 , but there is little evidence for eclipses. On the contrary, the EXOSAT observations by Culhane et al. (1990) showed clear primary and secondary eclipses in the $0.05-2.0 \mathrm{keV}$ band but not at higher energies. Pres et al. (1995) performed a detailed modeling of the EXOSAT light curve, finding that the emission region was located between the two stars. This scenario is not consistent with our observation, given the lack of significant eclipses and the observed modulation, which suggests the presence of active regions located on one or both stars which are visible between phases 0 and 0.5 .

Previous observations of CF Tuc at X-rays, EUV and radio wavelengths (Kürster \& Dennerl 1993; Schmitt et al. 1996; Gunn et al. 1997; Osten \& Brown 1999; Budding et al. 1999) have shown the clear presence of rotational modulation, stable over several orbital periods and anticorrelated with the optical light curve, indicating the presence of active regions located above starspot groups. On the contrary, we do not find any evidence for rotational modulation in our observation of CF Tuc. There might be an eclipse of the first flare, but the lack of data before the eclipse prevents any definitive conclusion.

Acknowledgements. We thank the referee for his comments and suggestions. This research was partially supported by Ministero dell'Istruzione, Università e Ricerca (MIUR) and by the Italian Space Agency (ASI).

\section{References}

Andersen, J., Clausen, J. V., Nordström, B., \& Reipurth, B. 1981, A\&A, 101, 7

Budding, E. 1985, IBVS, 2779, 1

Budding, E., Jones, K. L., Slee, O. B., \& Watson, L. 1999, MNRAS, 305, 966

Covino, S., Tagliaferri, G., Pallavicini, R., Mewe, R., \& Poretti, E. 2000, A\&A, 355, 681

Culhane, J. L., White, N. E., Shafer, R. A., \& Parmar, A. N. 1990, MNRAS, 243, 424

Dempsey, R. C., Linsky, J. L., Fleming, T. A., \& Schmitt, J. H. M. M. 1993, ApJS, 86, 599

Drake, S. A., Simon, T., \& Linsky, J. L. 1992, ApJS, 82, 311

ESA 1997, The Hipparcos and Tycho catalogues, ESA SP-1200

Favata, F., Mewe, R., Brickhouse, N. S., et al. 1997a, A\&A, 324, L37

Favata, F., Mineo, T., Parmar, A. N., \& Cusumano, G. 1997b, A\&A, 324, L41

Favata, F., Reale, F., Micela, G., et al. 2000, A\&A, 353, 987

Favata, F., \& Schmitt, J. H. M. M. 1999, A\&A, 350, 900

Fiore, F., Guainazzi, M., \& Grandi, P. 1999, Cookbook for NFI BeppoSAX Spectral Analysis v. 1.2, available at www.asdc.asi.it/bepposax/

Franciosini, E., Pallavicini, R., \& Tagliaferri, G. 2001, A\&A, 375, 196 Gehrels, N. 1986, ApJ, 303, 336

Güdel, M., Audard, M., Briggs, K., et al. 2001a, A\&A, 365, L336

Güdel, M., Audard, M., Magee, H., et al. 2001b, A\&A, 365, L344

Gunn, A. G., Migenes, V., Doyle, J. G., Spencer, R. E., \& Mathioudakis, M. 1997, MNRAS, 287, 199

Kürster, M., \& Dennerl, K. 1993, in Physics of Solar and Stellar Coronae, ed. J. F. Linsky, \& S. Serio (Dordrecht: Kluwer), 443 
Kürster, M., \& Schmitt, J. H. M. M. 1996, A\&A, 311, 211

Maggio, A., Favata, F., Peres, G., \& Sciortino, S. 1998, A\&A, 330, 139

Maggio, A., Pallavicini, R., Reale, F., \& Tagliaferri, G. 2000, A\&A, 356, 627

Mewe, R., Kaastra, J. S., \& Liedahl, D. A. 1995, Legacy, 6, 16

Mewe, R., Kaastra, J. S., van den Oord, G. H. J., Vink, J., \& Tawara, Y. 1997, A\&A, 320, 147

Mewe, R., Kaastra, J. S., White, S. M., \& Pallavicini, R. 1996, A\&A, 315, 170

Neff, J. E., Pagano, I., Rodonò, M., et al. 1996, A\&A, 310, 173

Ortolani, A., Maggio, A., Pallavicini, R., et al. 1997, A\&A, 325, 664

Osten, R. A., \& Brown, A. 1999, ApJ, 515, 746

Ottmann, R., \& Schmitt, J. H. M. M. 1996, A\&A, 307, 813

Pallavicini, R. 2001, in Cool Stars, Stellar Systems, and the Sun: 11th Cambridge Workshop, ed. R. G. Lopez, R. Rebolo, \& M. R. Zapaterio Osorio, ASP Conf. Ser. No. 223 (San Francisco: ASP), 377
Pallavicini, R., \& Tagliaferri, G. 1999, in Highlights in X-ray Astronomy, ed. B. Aschenbach \& M. J. Freyberg, MPE Rep., 272, 378

Paresce, F. 1984, AJ, 89, 1022

Pres, P., Siarkowski, M., \& Sylwester, J. 1995, MNRAS, 275, 43

Randich, S., Gratton, R., \& Pallavicini, R. 1993, A\&A, 273, 194

Schmitt, J. H. M. M., Stern, R. A., Drake, J. J., \& Kürster, M. 1996, ApJ, 464, 898

Tagliaferri, G., Covino, S., Cutispoto, G., \& Pallavicini, R. 1999, A\&A, 345, 514

Tagliaferri, G., Covino, S., Panzera, M. R., Pallavicini, R., \& Schmitt, J. H. M. M. 2001, in Cool Stars, Stellar Systems, and the Sun: 11th Cambridge Workshop, ed. R. G. Lopez, R. Rebolo, \& M. R. Zapaterio Osorio, ASP Conf. Ser. 223 (San Francisco: ASP), CD1177

Walter, F. M., \& Bowyer, S. 1981, ApJ, 245, 671

Walter, F. M., Cash, W., Charles, P. A., \& Bowyer, C. S. 1980, ApJ, 236, 212 